\title{
Die aktuelle Siedlungsentwicklung als Gestaltungsfaktor der Juralandschaft*
}

\section{Problemstellung}

Das Interesse an der Dynamik der Kulturlandschaft hat mich bewogen, die jurassische Kulturlandschaft nach ihrem jüngsten Wandel seit $1970 \mathrm{zu}$ untersuchen. Welches ist die Tendenz des räumlichen Wandels der siebziger Jahre, nachdem die vorausgegangenen Jahrzehnte vom Wirtschaftswachstum, einer Zunahme der Bevölkerung sowie einer starken Automobilisierung geprägt waren?

Hält dieser Trend weiterhin an und wie äußert er sich im Gesamtgefüge der Juralandschaft?

\section{Grundlagen}

In der Literatur sind Bedeutung und Methodik unserer Fragestellung für den Zeitraum der letzten 25 Jahre schon mehrfach behandelt worden (GALLUSSER 1970, 1977, 1980 und 1983). Bemerkenswert erscheint uns der Beitrag von TRACHSLER und ELSASSER 1983, worin die Daten beschrieben werden, welche in der Schweiz zur Untersuchung von Landnutzungsveränderungen zur Verfügung stehen. In Ergänzung zu den Angaben der vorstehenden Autoren stützen sich unsere Ermittlungen überdies auf die dynamisch aussagefähigen Nachführungsblätter der $\mathrm{L}+\mathrm{T}$ und auf die Grundbuch-Mutationsangaben ausgewählter Gemeinden.

Methodisch basiert unsere Arbeit auf den Daten der Volkszählungen 1970 und 1980 (Bevölkerung, Bewohnte Gebäude des Eidg. Amtes für Statistik). Sie sind in die Abb. 1 und 2 umgesetzt worden. Danach wurden die mutmaßlichen Beispielsgemeinden auf aktuellen Nachführungsblättern der Landestopographie und/oder Mutationsmeldungen bei Grundbuchgeometern überprüft und abschließend im Gelände auf Parzellenplänen kartiert. Als Grundlage für die

\footnotetext{
*An dieser Stelle verdanke ich den Herren G. Danielli, R. Leu und D.Wolfarth (Mitarbeiter am Geographischen Institut, Universität Basel) inre wertvolle Mithilfe bei der statistischen Aufbereitung bzw. bei den Geländearbeiten.

Dieser Vortrag wurde anlässlich des Symposiums "Die Landschaft des Jura" (Jahresversammlung der Naturforschenden Gesellschaft) am 14./15. Oktober 1983 in Porrentruy gehalten.
}

Berechnung der (ungefähren) Siedlungsfläche 1970 dienten z.T. die Angaben der "Arealstatistik der Schweiz» 1972.

Bei der Grundlagenbeschaffung halfen uns in verdankenswerter Weise: Gemeindekanzlei Olsberg, Gemeindekanzlei Welschenrohr, Herr J. Meier (Geometer, Delémont), Herr A. Auer (Geometer, Moutier), Herr J.C.Stotzer (Bundesamt für Landestopographie, Bern).

\section{3. Übersicht über die allgemeine Dynamik}

Wir sehen die räumlichen Veränderungen des Juras im generellen Zusammenhang mit den externen Entwicklungen in Wirtschaft und Gesellschaft. Vor allem hat sich mit dem Kanton Jura seit 1979 eine neue politische Raumeinheit herausgebildet und damit eine neue mögliche Variante der Raumentwicklung. Dazu ist die ökonomische Konjunktur von einer zunehmenden Depression der regionalen Wirtschaft, vor allem der Uhrenbranche, abgelöst worden. Als drittes beginnt sich auch im Jura das Lenkungsinstrument der Raumplanung allmählich auszuwirken, ebenso die verschiedenen Formen der Rurbanisierung, wie ich sie 1977 für dieses Gebiet der Schweiz dargelegt hatte.

Betrachten wir zuerst die Bevölkerungsdynamik auf Grund der Volkszählung 1970 und 1980, wie sie auf Abb. 1 zu ersehen ist. Das Resultat der Bevölkerungsentwicklung 1970-80 für die 488 Juragemeinden ist erstaunlich: praktisch Nullwachstum! Wie Abb. $1 \mathrm{zu}$ erkennen gibt, steht dem flächenhaft dominierenden Bevölkerungsrückgang ein beschränktes Wachstum in den Randlagen der Verstädterungsräume von Yverdon, Neuchâtel, Delémont, Biel und Aarau gegenüber. Dazu zeichnen sich noch die Verstädterungsachse Basel-Hochrhein sowie ihre Ausläufer N2 und Fricktal durch mäßige Zunahmen aus. Eingeordnet in die vorerwähnte Wirtschafts- und Sozialdynamik widerspiegelt die Bevölkerungsentwicklung insgesamt einen Rückgang der natürlichen Reproduktion und eine breite Abwanderung in die Verstädterungszonen.

Werner Gallusser, Prof., Dr., Geographisches Institut der Universität Basel, Klingelbergstr. 16, 4056 Basel 

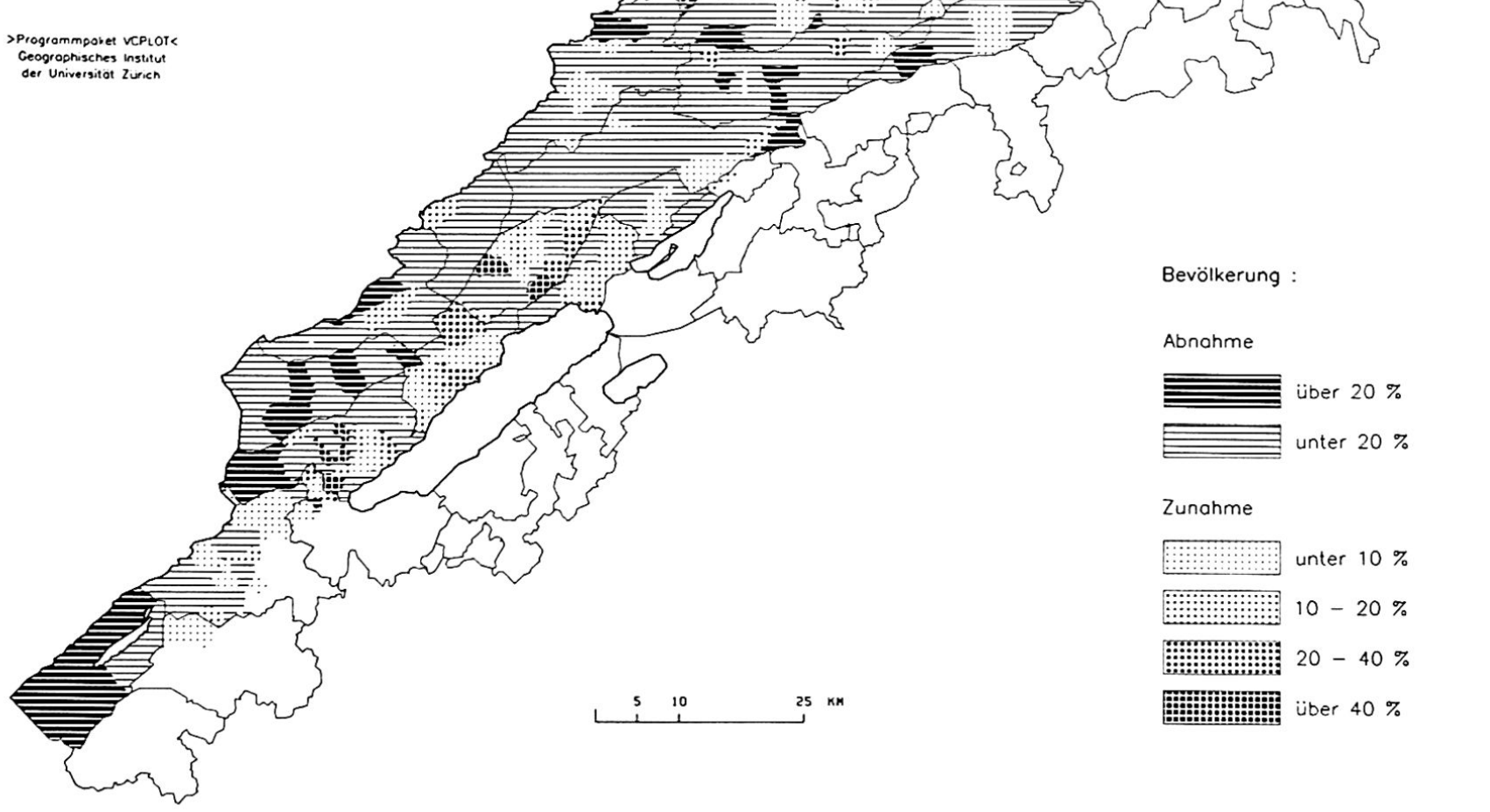

Abb.1 Bevölkerungsentwicklung 1970-80 (Quelle: Bundesamt für Statistik)

Wie äußert sich nun dieser generelle Tatbestand der Bevölkerungsdynamik im Bereich des Siedlungsraumes? Bestimmt der Schlüsselindikator der Kulturlandschaft, der Mensch in seiner Bevölkerungszahl, die räumliche Entwicklung seiner Siedlung? Wenn dem so wäre, müsste unsere Abb. 2 die Entwicklung der «Bewohnten Häusen» auf die vorgenannten Ballungsräume der Bevölkerung konzentriert zeigen. Dies trifft jedoch nur bedingt zu: die Zunahme des Wohnraums ist breit gestreut, gewiß in den urbanen Randzonen mit höchsten Werten, aber auch in den Ruralräumen von beachtlichem Ausmaß, so da $\beta$ im ganzen Jura doch ein mittlerer Zuwachs von $16,2 \%$ an Wohnhäusern zu verzeichnen ist.

\section{Typen der aktuellen Siedlungsdynamik im Jura}

Es sei festgehalten, da $\beta$ die jurassischen Siedlungen zwischen 1970 und 80 trotz Stagnation der Bevölkerungszahl an Wohnraum um ein Sechstel zugenommen haben. Und wir fragen uns, unter welchen Hauptumständen und wo sich dieser Zuwachs abgespielt hat.
Auf Grund der Bevölkerungszahlen könnte eine Zuordnungsmatrix zwischen Bevölkerungs- und Siedlungsdynamik erstellt und daraus eine vereinfachte Typologie der kulturlandschaftlichen Dynamik abgeleitet werden. Dieser Typologieversuch scheint mir geographisch jedoch nur unter der Prämisse sinnvoll, daß die bewohnten Gebäude in ihrem Bezug zur Bevölkerungsentwicklung aussagefähige Kennzeichen der gesamten Kulturlandschaft darstellen, was a priori postuliert sei.

Im Sinne einer methodisch-praktischen Einschränkung betrachten wir im Folgenden vor allem jene zwiespältige Raumdynamik ausführlicher, welche bei abnehmender Bevölkerung eine wesentliche Zunahme des Siedlungs-Wohnraums gezeitigt hat.

Vorerst verdient aber gleichwohl der stagnierende Gemeindetyp (mit Abnahme oder Stagnation von Bevölkerung und Siedlung) unsere Beachtung insofern, als ihm eine vieldeutige Dynamik innewohnt. Beim Stagnationstyp" darf nicht unbedingt ein Erlöschen der kulturräumlichen Aktivitäten erwartet werden; so zeigt etwa das Beispiel Welschenrohr SO, daß sich die Wirtschaftsförderungskommission intensiv um Ersatzbetriebe für den 1975 erfolgten Zusammen- 


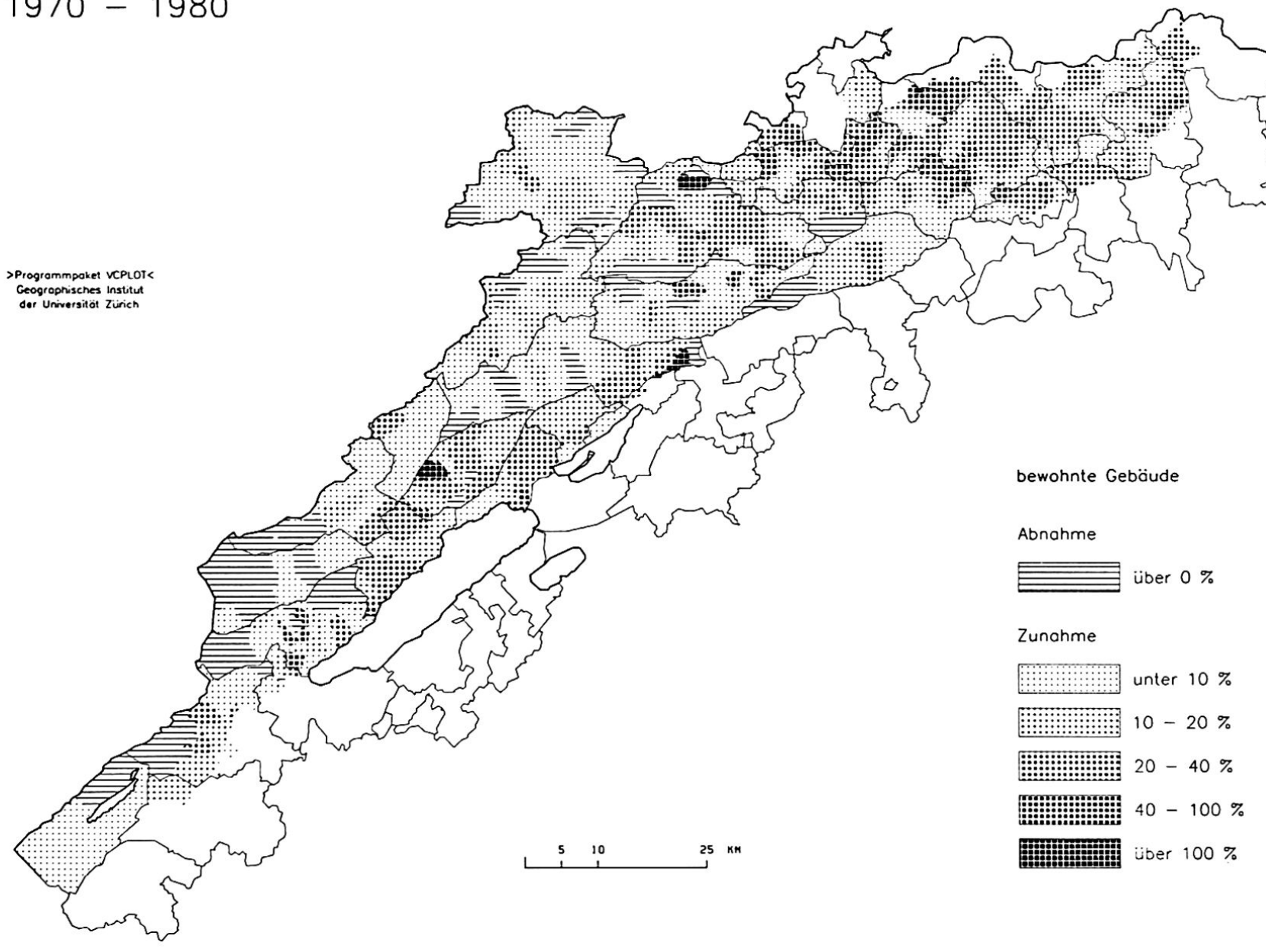

Abb. 2 Siedlungsdynamik 1970-80 (“Bewohnte Gebäude»; Quelle: Bundesamt für Statistik)

bruch in der Uhrenbranche bemüht. Es werden hier z.T. mit Erfolg leerstehende Fabrikgebäude durch Neubetriebe diverser Branchen genutzt. Leerstellung und Folgenutzung von Gebäuden, zunehmende Pendelarbeit und - nach den Tiefpunkten von 1977 und 1978 - eine zaghafte Wiederaufnahme des Wohnungsbaus lassen sich nicht aus den nüchternen Pauschalzahlen der Schweizer Statistik herauslesen. Stagnationsgemeinden, seien sie nun im Hochjura oder am Jurasüdfuß lokalisiert, verdienten auch aus entwicklungspolitischer Sicht eine spezielle Beachtung durch die Geographie.

Es sei ergänzend darauf verwiesen, daß es auch andernorts in Juratälern zu bemerkenswerten Stagnationen kommen kann, zur Abwanderung von Pendlern und zur Leerstellung von Gebäuden. Beispiele hierfür wären etwa Soyhières JU und Frinvillier (BE, an der T6), wo Industrie- oder Verkehrsimmissionen die Wohnlichkeit beeinträchtigen und als Push-Faktoren wirken.

Betrachtet man die aktuelle Siedlungsentwicklung im Jura als räumliche Gesamterscheinung, so können unter den vorgenannten Einschränkungen drei Typen modellhaft herausgestellt werden, und zwar bei einer regional stagnierenden Bevölkerung:

1. Die Verstädterungsrand-Besiedlung

2. Die Jurahang-Besiedlung und

3. Die Freiland- und Höhen-Besiedlung (Ferienhäuser).

\subsection{Die Verstädterungsrand-Besiedlung}

Sie hat die Außengebiete der Jura- und der JurarandStädte ergriffen und beschert insbesondere ländlichen Kleingemeinden in günstiger Kontaktlage eine rasche Überbauung der relativ überschaubaren Bauzonen. Hiezu sei etwa auf die Beispiele Olsberg AG, Rossemaison JU und Les Hauts Geneveys NE verwiesen. Man kann diese Siedlungsart in den Zusammenhang der städtischen Kern-Rand-Wanderung stellen. Auch im Jura der 1970er Jahre schreitet die städtisch aktive Lebensform voran und überträgt sich - mit einem höheren Raumbedarf pro Kopf - in die bisher ländliche Stadtperipherie. Zwar vergrößert sich die Ein- 


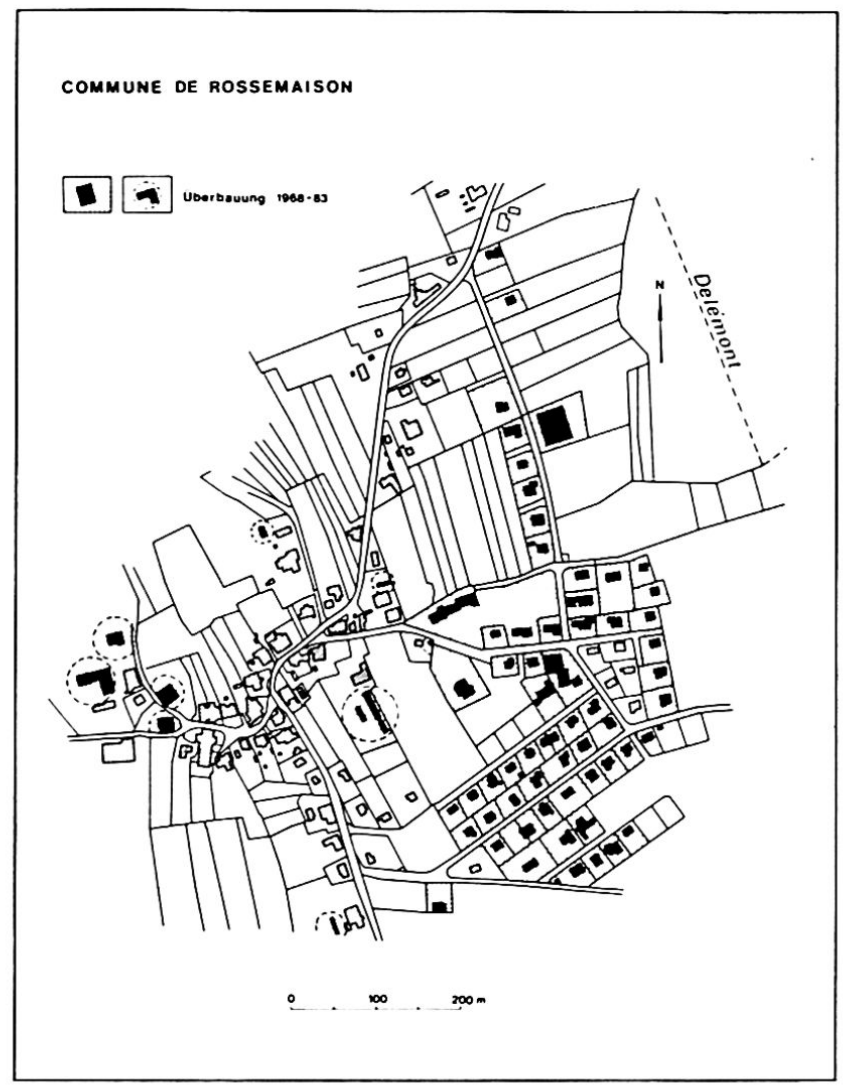

Abb. 3 "Verstädterungsrand-Besiedlung" 1968-83 in Rossemaison JU

wohnerzahl in der Regel, jedoch auf Kosten der benachbarten Stadtkern-Gemeinden. Am Beispiel Rossemaison ersieht man die beträchtliche Flächendynamik der letzten 15 Jahre: verglichen mit dem Siedlungsraumzuwachs zwischen 1952 und 1968, bei welchem etwa 1,30 ha überbaut worden war, steigerte sich die Überbauung zwischen 1968 und 1983 mit über 40 Neubauten auf das Dreifache (vgl. Abb. 3); die Bevölkerung wuchs dagegen zwischen 1970 und 1980 um 35\%. Ähnliches wäre über den Aargauer Klosterweiler Olsberg zu berichten, wo 1970 bis 1983 ein Siedlungsraumzuwachs von $89 \%$ verzeichnet werden konnte. Das benachbarte Magden zeigte 1970 bis 1980 eine ebenfalls markante Zuwachsquote von $55 \%$.

\subsection{Die Jurahang-Besiedlung}

Sie vollzieht sich im zentralen Kettenjura seit Jahren mit zunehmender Prägung des Landschaftsbildes und erfaßt die etwas abseitigen Dörfer mit gutem Straßenanschluß, wie z.B. Liesberg, Dittingen (Abb.4), Perrefitte und Vauffelin (alle im Kanton Bern); diese Gemeinden weisen alle gut besonnte Hänge auf.

Das Hanggelände, bisher meist als Weideland oder Naturwiese genutzt, erlangt als aussichtsreiche Wohnlage eine Neubewertung durch Bauinteressenten. Das sind einheimische Pendler oder Auswärtige, welche

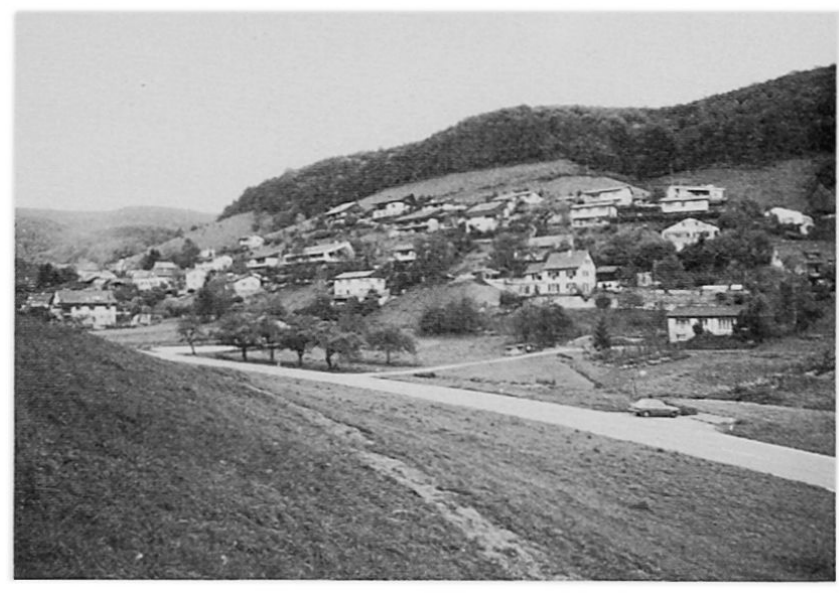

Abb. 4 "Jurahang-Besiedlung" in Dittingen BE. 1983 (Foto: Arn. Bühler, Geogr. Inst. Basel)

die längeren Pendeldistanzen wegen der guten Wohnqualität in Kauf nehmen. Die Jurahang-Besiedlung verläuft hektisch und physiognomisch auffällig. Sie verwandelt ehemalige Gemeindeweiden (z. B. Liesberg), Wässerwiesen (Dittingen) und Juramatten in relativ schwach bevölkerte Einfamilienhaus-Zonen mit aufwendigen Erschließungsarbeiten und einem permanenten Motorfahrzeugverkehr. Seit 1970 wurden z.B. in der Gemeinde Vauffelin rund 2,4 ha Jurahang $(=24 \%$ der gesamten Neusiedlungsfläche von 1970) beansprucht.

\subsection{Die Freiland- und Höhen-Besiedlung}

Dieser dritte Siedlungstyp hat die noch entlegeneren Juragebiete erfaßt, d.h. die Gebiete in den dörflichen Außenfluren und die Höhenstufe der waldarmen Bergrücken und Plateaus. Als Testgemeinden wurden vor allem Movelier JU, aber auch Champoz und Vauffelin BE untersucht. Die Freiland- und HöhenBesiedlung ist eine eigentliche Infiltrationsbewegung von verschiedenartigsten "Freizeitgehäusen», vom finnischen Blockhaus und dem Berner Chalet zum Colonial Bungalow mit Rasen bis zur Do-it-yourselfBretterbude und dem festgemauerten Campingwagen... Es war und ist ein Wettlauf um die besten und billigsten Parzellen irgendwo in der Juralandschaft: sie müssen nur mit dem Auto zugänglich sein! Gerade dieser dynamische Typ der jurassischen Siedlungslandschaft verdient unsere besondere Beachtung. Schon 1970 und 1977 haben wir in speziellen Studien über die NW-Schweiz und den Jura darauf hingewiesen und möchten es im Blick auf Movelier (Abb. 5) wiederholen: die damals festgestellte Rurbanisierung geht weiter. Ergänzend zu den statistischen Befunden zeigte unsere Geländeuntersuchung, da $\beta$ sich die Neusiedlungsfläche $1970-83$ weiterhin um $77 \%$ vergröBert hat; in diesem Zeitraum wurden 94 Gebäude (inkl. Garagen) neu errichtet. Das traditionelle Dorf 


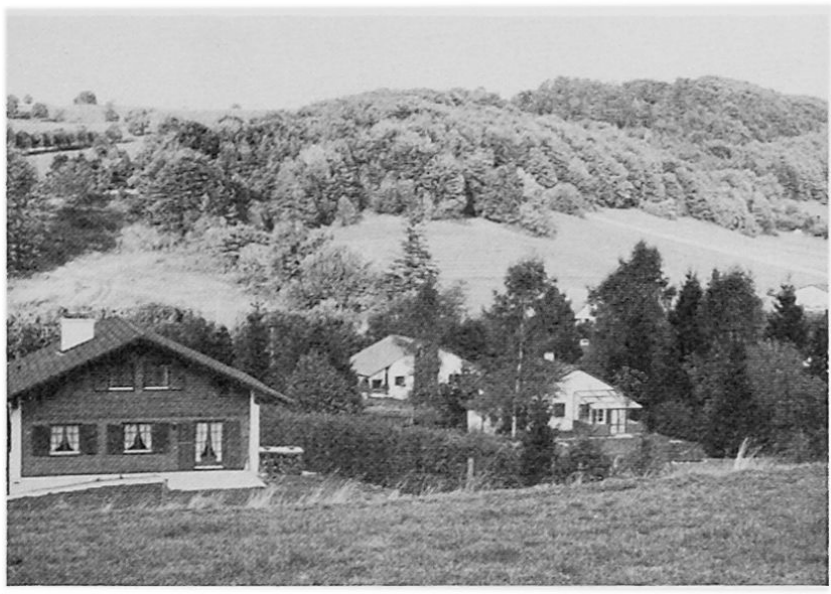

Abb. 5 "Freiland- und Höhen-Besiedlung" in Movelier JU. 1983 (Foto Arn. Bühler, Geogr. Inst. Basel)

auf dem flachen Jurarücken (700 m ü. M.) hat nun durch den hektischen Ferienhaus-Bau seine feste Kontur verloren; damit sind auch beträchtliche Teile des Freilandes in ein unregelmäßiges Siedlungsmosaik einbezogen und einer städtisch-intensiveren Beanspruchung zugeführt worden.

Ähnliche Besiedlungen lassen sich auch in den Freibergen, z. B. Cerneux-Veusil ( $1070 \mathrm{~m}$ ü. M., JU) und auf den Prés de la Montagne (Gemeinde Vauffelin BE, $1000 \mathrm{~m}$ ü. M.) wie auf dem Moron ( $1100 \mathrm{~m}$ ü. M., Champoz BE) feststellen. Es ist zu beachten, daß sich dieser Besiedlungstyp trotz baugesetzlichen Vorschriften im Jura noch immer durchzusetzen vermag, da seine naturnahe Landschaft auf den motorisierten Landsucher offenbar weiterhin als unwiderstehlicher Pull-Faktor wirkt, und weil die negative Gesamtwirkung einer derartigen Besiedlung noch zu wenig deutlich gesehen wird. Muß auch hier auf das Waldsterben hingewiesen werden, um die schleichenden Auswirkungen einer auf Automobilismus beruhenden Besiedlung der Jurahöhen zu verdeutlichen? Auch nach den tourismus-ökonomischen Befunden von RUMLEY (1983) verläuft die jurassische Zweithaus-Bauentwicklung, welche praktisch der Freiland- und Höhen-Besiedlung entspricht, vielerorts zu extrem. Die überbordenden FerienhausKapazitäten einzelner Gemeinden (mit bis $40 \%$ des Übernachtungs-Totals) verunmöglichen auch eine regionalwirtschaftlich vertretbare Erholungsnutzung.

\section{Zusammenfassung}

1. Der Schweizer Jura zeigt von 1970 bis 80 eine stagnierende Bevölkerungszahl, wobei sich neben ausgedehnten «Entleerungsräumen» einige «Ballungsgebiete» im Bereich der Regionalzentren abzeichnen.
2. Diese demographischen Bewegungen sind mit spezifischen Siedlungsvorgängen gekoppelt bzw. von anders gerichteten Siedlungsbewegungen überlagert.

3. Vor allem verlagert sich die Bevölkerung in der Verstädterungsrand-Besiedlung an die Peripherie der jurassischen Stadtgebiete, wo große Wohnareale zonengemä $\beta$ besiedelt werden. Die damit verbundene Sozialdynamik erfaßt die Kleingemeinden besonders stark, dazu werden die landwirtschaftlichen Vorzugsflächen durch diese unmittelbare Verstädterung am nachhaltigsten geschmälert.

4. Die reine Jurahang-Besiedlung hat sich vor allem in entlegenen Pendlergemeinden des zentralen Kettenjuras entwickelt. Sie hat günstig exponierte Jurahänge, meist Extensivflächen der Landwirtschaft, in uniforme Einfamilienhaus-Zonen verwandelt. Dieser Besiedlungstyp ist gleichzeitig Ausdruck sozialer Entmischungsvorgänge im Zeichen des Pendelverkehrs und der Verdrängung extensiver Agrarformen. Als ein aktiver Rurbanisierungsprozeß gestaltet die gegenwärtige Jurahang-Besiedlung die Landschaft physiognomisch und ökologisch tiefgreifend um.

5. Die Freiland- und Höhen-Besiedlung bewirkt die Infiltration der dörflichen Außenfluren und der Jurahöhen mit Ferienhäusern, Autoverkehr und auswärtigen Besuchern. Obschon die Freiland- und Höhen-Besiedlung als extensive Rurbanisierung Nutzungskombinationen zwischen Erholung, Berglandwirtschaft, Forstwirtschaft und Landschaftsschutz denkbar erscheinen läßt, verlangt dieser Eingriff in den wohl natürlichsten Bereich der Juralandschaft nach strengerer Handhabung einer umweltbewußten Raumplanung, zumindest auch über die Kantonsgrenzen hinweg.

\section{Literatur}

AREALSTATISTIK DER SCHWEIZ. Statist. Quellenwerke der Schweiz, H.488. Bern 1972

GALLUSSER, W. A. (1970): Struktur und Entwicklung ländlicher Räume der NW-Schweiz. Basler Beiträge zur Geographie 11.

GALLUSSER, W.A. (1978): Der Nordwestschweizer Jura im Wandel der Gegenwart - eine humangeographische Bilanz. In: Plan 35/11, 30-34.

GALLUSSER, W. A. (1980): Remarques méthodologiques sur la représentation de la dynamique de l'environnement, basée sur des cartes de la propriété foncière. In: Actes du Symposium internat. sur la Cartographie de l'Environnement et de sa dynamique, Caen, 23-35.

RUMLEY, P.A. (1983): Le tourisme jurassien. In: Geographica Helvetica 38/2, 73-77.

TRACHSLER, H. und ELSASSER, H. (1983): Landnutzungsveränderungen in der Schweiz. In: Vermessung, Photogrammetrie, Kulturtechnik 1983/2, 29-34. 